\title{
Absorption-desorption phase transition induced by parametric modulation
}

\author{
C. Van den Broeck \\ Limburgs Universitair Centrum, B-3590 Diepenbeek, Belgium \\ R. Kawai \\ University of Alabama at Birmingham, Birmingham, Alabama 35294
}

(Received 23 October 1997)

\begin{abstract}
We present a nonequilibrium absorption-desorption phase transition, induced by a time-periodic parametric modulation, and compare it with the phase transitions induced by white and dichotomous Markov noise. In the case of time-periodic modulation and dichotomous Markov noise, the phase transition is found to be reentrant with respect to the strength of the spatial coupling. [S1063-651X(98)02804-9]
\end{abstract}

PACS number(s): 05.40. $+\mathrm{j}$

\section{INTRODUCTION}

In a number of recent papers [1-20], several intriguing cooperative phenomena have been reported in spatially distributed systems subject to state-dependent noise. Genuine nonequilibrium phase transitions of both first and second order and involving the breaking of the symmetry of the evolution equations or of time and space translation invariance have been presented. Models with state-dependent noise have also appeared in several other areas of physics, including lasers [21], hydrodynamics [22], and growth phenomena [23]. The purpose of this paper is to investigate the effect of periodic modulation with disorder in one of the simpler models, exhibiting a phase transition out of an absorbing state. The linearity of the model allows one to obtain a general exact solution of the mean-field version. For comparison we also include the result for a perturbation by white noise and dichotomous Markov noise and numerical results for dimensions $d=1$ and 2 . The study reveals that periodic perturbation with disorder can give rise to phase transitions that are similar to those induced by noise. At the same time, we clarify the rather surprising mechanism behind such modulation-induced phase transitions by tracing it back to the (transient) increase of the first moment [16]. In fact, the system seems to escape out of the attracting state by pulling on a periodically changing pool of transiently escaping members. The balance of coupling between those sites that are on return to the absorbing state and those that are on a transient escape is, however, delicate and a reentrant phase transition back to the absorbing state is observed for large coupling strengths.

\section{MODEL}

We first consider a single scalar variable, decaying at a rate that is parametrically modulated:

$$
\dot{x}(t)=[-1+\xi(t)] x(t) .
$$

This equation has the solution

$$
x(t)=\exp \left[-t+\int_{0}^{t} \xi(\tau) d \tau\right] x(0) .
$$

We will focus on three types of parametric modulation, namely, (a) $\xi(t)$ being a realization of Gaussian white noise [24], with $\langle\xi(t)\rangle=0$ and $\left\langle\xi(t) \xi\left(t^{\prime}\right)\right\rangle=\sigma^{2} \delta\left(t-t^{\prime}\right)$; (b) $\xi(t)$ corresponding to a dichotomous Markov process [24], with $\xi(t)= \pm \Delta$ and switching rate $k$; and (c) a time-periodic perturbation $\xi(t)=A \cos (\omega t+\varphi)$ with a phase $\varphi$ chosen at random in $[0,2 \pi]$.

Our main interest is in case (c), for which one easily verifies that $x(t) \rightarrow 0$ for all values of the amplitude, phase, and frequency, except for case of quenched disorder $\omega=0$ with $A>1$. In cases (a) and (b), this property remains true, at least with probability one. In other words, the absorbing state $x=0$ is a global attractor. However, the first moment $\langle x(t)\rangle$, which is given by (a) $\langle x(t)\rangle=e^{-t} e^{\left(\sigma^{2} / 2\right) t} x(0)$, (b) $\langle x(t)\rangle=e^{-t}\left(\lambda_{+} e^{\lambda_{-} t}-\lambda_{-} e^{\lambda_{+} t}\right) /\left(\lambda_{+}-\lambda_{-}\right) x(0)$, where $\lambda_{ \pm}=-k \pm \sqrt{k^{2}+\Delta^{2}}$; and (c) $\langle x(t)\rangle=e^{-t} I_{0}[(2 A / \omega) \sin (\omega t /$ 2) $] x(0)$, where $I_{0}$ is the zeroth-order first kind of modified Bessel function, diverges for $t \rightarrow \infty$ when (a) $\sigma^{2}>2$, (b) $\Delta^{2}>2 k+1$, and (c) $A>1$ for $\omega=0$. For $\omega \neq 0$, or in the presence of an additional nonlinear saturation term $-x^{3}$ on the right-hand side of Eq. (1), the divergence of $\langle x\rangle$ is removed, but a transient increase of $\langle x(t)\rangle$ above its original value is possible. Note also that in cases (b) and (c) the increase is preceded by a decrease for small times; see Fig. 1

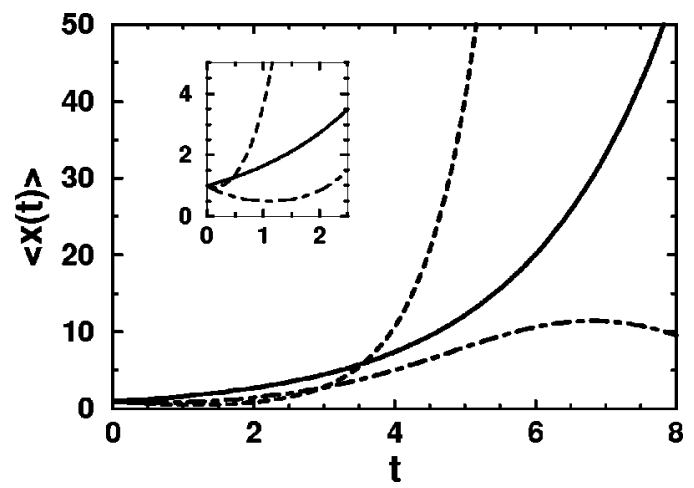

FIG. 1. Time evolution of the first moment for the cases of Gaussian white noise (solid line, $\sigma^{2}=3$ ), dichotomous noise (dashed line, $\kappa=0.1$ and $\Delta=1.5$ ), and the periodic modulation (dotdashed line, $\omega=1$ and $A=4$ ). The inset shows the detailed early evolution. 
for some typical trajectories. The origin of the increase or divergence can be explained most easily for the case of Gaussian white noise. From Eq. (1) it follows that $\ln x(t)+t=\int_{0}^{t} \xi(\tau) d \tau$, so that $\ln x(t)+t$ is ordinary Brownian motion with the diffusion coefficient equal to $\sigma^{2} / 2$. We conclude that the probability density $P(x, t)$, with initial condition $P(x, t=0)=\delta\left(x-x_{0}\right)$, is given by

$$
P(x, t)=\frac{\exp \left[-\frac{\left(\ln x+t-\ln x_{0}\right)^{2}}{2 \sigma^{2} t}\right]}{x \sqrt{2 \pi \sigma^{2} t}} .
$$

From this explicit result, one clearly sees that $P(x, t) \underset{t \rightarrow \infty}{\longrightarrow} \delta(x)$, while the development of a long tail in $x$ leads to a divergence of $\langle x(t)\rangle$ for $\sigma^{2}>2$. The increase or divergence of the first moment thus appears to be a mathematical peculiarity resulting from those realizations of the noise that lead to extremely large excursions away from zero. We will show below, however, that these realizations are the driving force behind genuine modulation-induced phase transitions.

\section{MEAN-FIELD DESCRIPTION}

Our main interest is to couple a set of $N$ elements $x_{i}$, each of which obeys the evolution equation (1). The intuitive idea is that the global behavior of these elements, which are subject to distinct realizations of the disorder or noise, is influenced more by those that tend to destabilize the reference state, even if they are in the minority, than by the more common typical behavior of the large majority. The fact that the first moment undergoes an increase above its initial value, even when this is only temporarily so as in case (c), was suggested in [16] as the mechanism behind the phase transition in the spatially coupled version of the system.

We consider only the simple case of harmonic coupling, namely,

$$
\dot{x}_{i}=\left[-1+\xi_{i}(t)\right] x_{i}-\sum_{j} K_{i j}\left(x_{i}-x_{j}\right)
$$

Furthermore, the elements are typically arranged on a regular lattice, e.g., a cubic lattice in dimension $d$, and the coupling is nearest neighbor with strength $K_{i j}=K / 2 d$. For simplicity, we will also assume that the realizations of the process $\xi_{i}(t)$ are independent (random phase and white noise in space). Even though Eq. (4) is linear and the equations for its moments are closed, an exact solution for the probability density could not been found. In fact, in case (c), Eq. (4) is completely deterministic and a detailed discussion of the resulting dynamic properties is probably quite complicated. A rather clear picture is available, however, for the case of Gaussian white noise $[1,8,13,25]$. In particular, there exists a sharp threshold at which the system will stop converging to the absorbing state $x_{i}=0 \forall i$ [26], but the exact location of this transition is only known in the limit of infinitely strong coupling $[1,7,8]$. In order to obtain exact analytic results including the cases of the other types of modulation, we turn to a mean-field description $[6,27,28]$. Numerical results for finite-dimensional system will be presented below. The

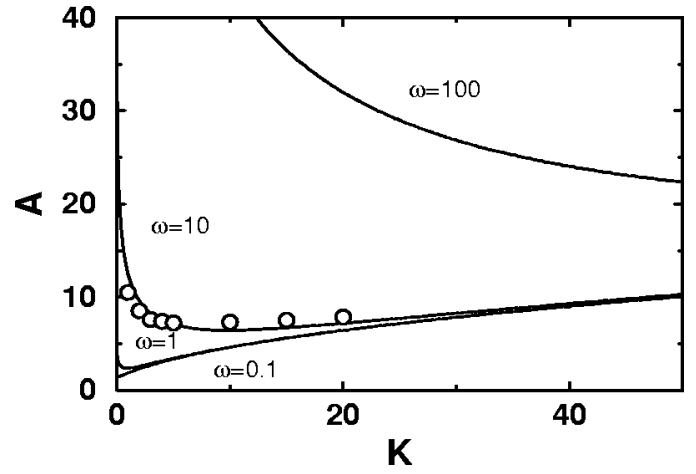

FIG. 2. Phase diagram: the lines indicate the boundary of absorbing and desorbing phases obtained from the mean-field theory for case (c) of periodic modulation ( $\omega=0.1,1,10$, and 100). Circles represent the results of computer simulation for the onedimensional system $(\omega=10)$.

mean-field description can be realized by considering global coupling, $K_{i j}=K / N$ for all pairs $i, j$, and taking the thermodynamic limit $N \rightarrow \infty$. Equation (4) transforms into the meanfield equation

$$
\dot{x}=[-1+\xi(t)] x-K(x-\langle x\rangle),
$$

in which the subscript $i$ has been dropped for simplicity of notation. We stress that, as a result of the law of large numbers, one expects $\langle x(t)\rangle=\lim _{N \rightarrow \infty} 1 / N \sum_{j=1}^{N} x_{j}(t)$ to be a selfaveraging macroscopic intensive variable (see the Appendix). Equation (5) can be solved exactly:

$$
\begin{aligned}
x(t)= & \exp \left[-(1+K) t+\int_{0}^{t} \xi(\tau) d \tau\right] x(0)+\int_{0}^{t} d \tau \exp [-(1 \\
& \left.+K) \tau+\int_{0}^{\tau} \xi\left(\tau^{\prime}\right) d \tau^{\prime}\right] K\langle x(t-\tau)\rangle
\end{aligned}
$$

and the self-consistent value of $\langle x(t)\rangle$ can be easily obtained from either Eq. (5) or (6). The line in the phase diagram separating the absorption regime $\langle x(t)\rangle \rightarrow 0$ from the explosion regime $\langle x(t)\rangle \rightarrow \infty$ is then found to be (a) $\sigma^{2}=2$ (independent of $K$; in fact, the result for $\langle x(t)\rangle$ is identical to that for $K=0$ ), (b) $\Delta^{2}=1+2 k+K$ (but this is the line of the reentrant transition; explosive behavior is observed for coupling constants smaller than this value of $K$ ), and (c) $\int_{0}^{\infty} d t K \exp [-(1+K) t] I_{0}[(2 A / \omega) \sin (\omega t / 2)]=1$ and is represented in Fig. 2.

\section{DISCUSSION}

For $K \rightarrow 0$, the onset of explosion coincides, as expected, with the parameter values at which $\langle x\rangle$ diverges in the uncoupled case $K=0$. In particular, no explosion at all occurs for case (c) of periodic modulation. However, with increasing value of $K$, the properties of the coupled and uncoupled system become quite different. We start with a discussion of the behavior for case (a) of Gaussian white noise. In the uncoupled system, the divergence of $\langle x(t)\rangle$ for $\sigma^{2}>2$ corresponds to a highly irregular spatiotemporal intermittent behavior, with fewer and fewer units making ever larger excur- 


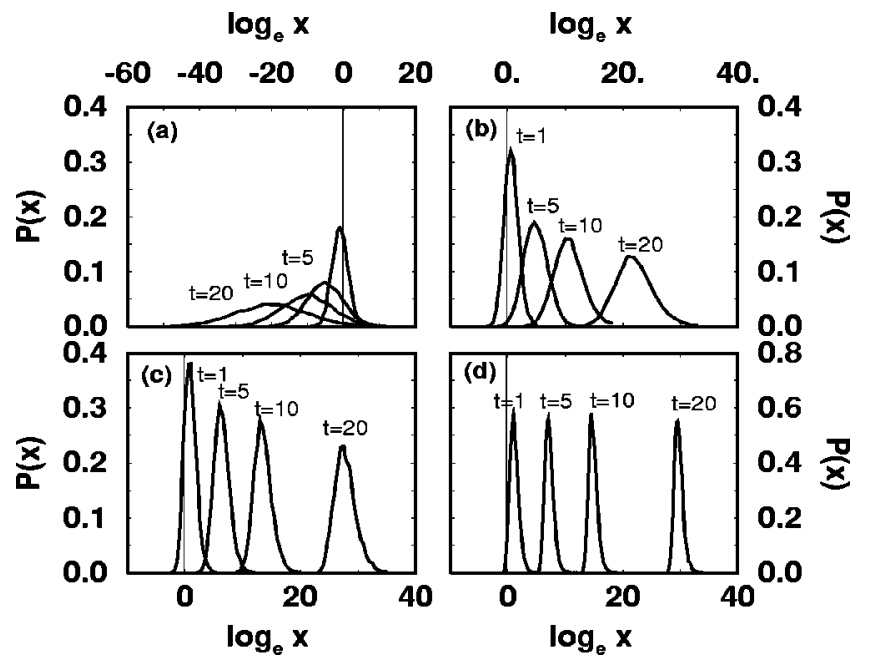

FIG. 3. Time evolution of the probability density for the case of Gaussian white noise $\left(\sigma^{2}=5\right)$ : (a) no coupling, (b) one-dimensional coupling, (c) two-dimensional coupling, and (d) mean-field coupling. A coupling strength $K=3$ is used for the coupled systems.

sions away from zero, while the large majority of elements is rapidly converging to zero. The behavior is quite different in the globally coupled system, when the coupling constant is sufficiently strong. This is illustrated in Fig. 3, where we represent the probability density $P(x, t)$ of a single unit to take on the value $x$ at time $t$, for the uncoupled and the mean-field coupled system. For comparison we have also included the corresponding results for dimensions $d=1$ and 2. Note that the time dependence of the first moment $\langle x\rangle$ is exactly the same in all four cases. For $\sigma^{2}>2$, it diverges exponentially fast. However, $P(x, t)$ converges to a $\delta$ function at zero in the uncoupled case, in perfect agreement of course with the discussion given above. When the units are coupled with a large enough coupling constant $K>\sigma^{2} / 2$ (see the Appendix), this is no longer the case and the time dependence of the first moment reflects much more closely the global behavior of $P(x, t)$. In fact, the situation is more subtle if higher-order moments are considered (see [29] and the discussion in the Appendix): Some kind of spatiotemporal intermittency still shows up, with the behavior of such moments dominated by rare, localized spikes of explosion. Note, however, that this intermittent behavior is peculiar to the linear model and disappears in the presence of nonlinearities. One concludes that in the white-noise case, the effect of the coupling is as follows: The sites, at which unlikely realizations of the noise lead to explosive behavior, pull the other sites away from the absorbing state. As the coupling increases, the behaviors of all the sites become more and more alike and intermittency is reduced. It is, however, never completely eliminated (except if a nonlinear saturating term is added) and a reentrant to the absorbing state for sufficiently strong coupling is absent.

For case (b) of the dichotomous Markov process, the behavior is quite similar, with, however, the important difference that there is a reentrant transition with respect to the coupling constant $K$. This phenomenon, which is quite unlike what one finds in equilibrium phase transitions, also has been observed recently in another model [7] subject to multiplicative colored noise [30].
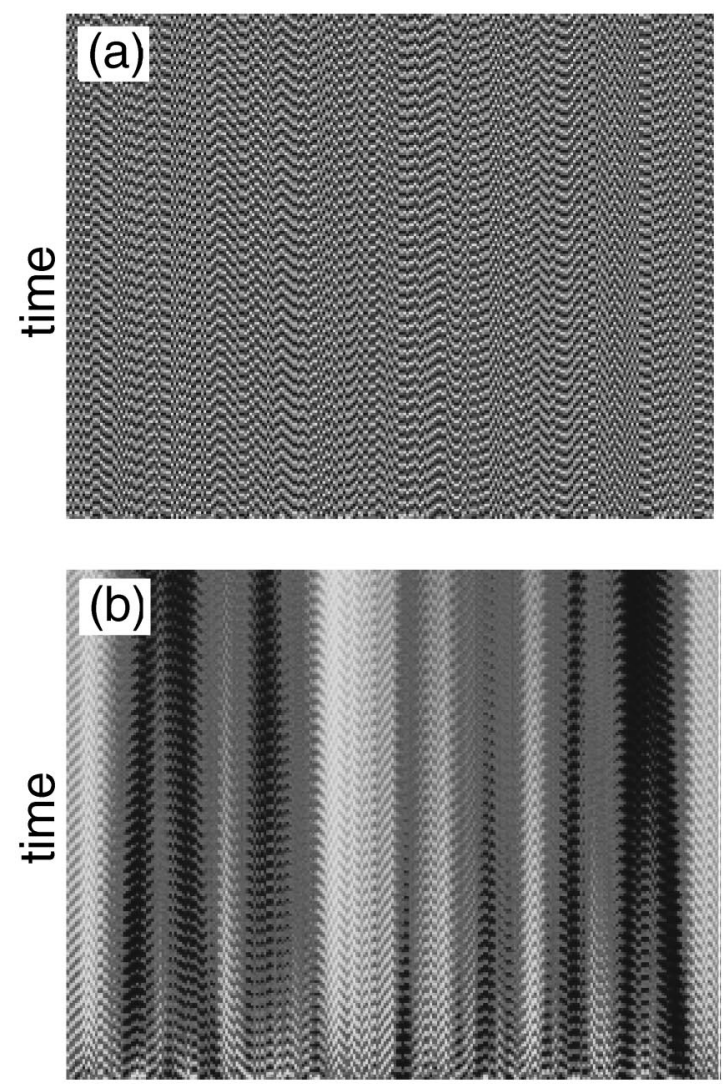

space

FIG. 4. Time evolution of individual sites. A system of 256 sites with a periodic modulation $(K=5, \omega=10$, and $A=10)$ evolves for $t=10$ units. Gray-level coding identifying the sites (horizontal axis) with the larger- $x$ values (light color) versus the smaller- $x$ values (dark color) as a function of time (vertical axis). Results are shown for (a) mean-field coupling and (b) one-dimensional coupling.

We finally turn to the most interesting case (c) of periodic modulation. It provides a more dramatic and convincing example of our statement that those realizations of disorder that are responsible for the transient increase of the first moment in the uncoupled case can completely destabilize the macroscopic system. Indeed, one notes that for a sufficiently large value of the amplitude $A$, the $\langle x\rangle=0$ state becomes unstable as one crosses a critical value of the coupling constant $K$ and the system exhibits a nonequilibrium phase transition towards explosive behavior. This fact is even more remarkable if one remembers that every single element on its own experiences a fast exponential return to the absorbing state $x=0$. Apparently, those members that experience a transient increase in their $x$ values pull over the other sites away from zero. However, as those members start relaxing, other sites take over and pull the system even further away from zero, and so on, ad infinitum. In other words, the system seems to be pulling itself up at a periodically changing pool of transiently growing members. This can be quite nicely illustrated by identifying which sites have the larger- and smaller- $x$ values as a function of time; see Fig. 4. Note the difference between the mean field and the $d=1$ situation. In the latter case, the larger- $x$ values stay more or less concentrated on the same sites with time, while a slow, nucleationlike process 

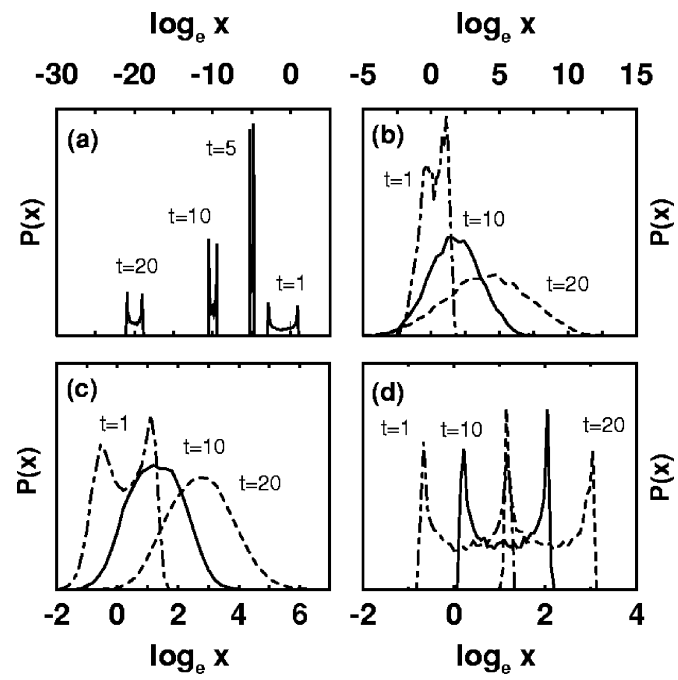

FIG. 5. Time evolution of the probability density for the case of periodic modulation: (a) no coupling, (b) one-dimensional coupling, (c) two-dimensional coupling, and (d) mean-field coupling. A coupling strength $K=2$ is used for the coupled systems.

takes place that joins together neighboring larger- and smaller- $x$ values, respectively. The resulting behavior for the single-site probability density $P(x, t)$ are shown in Fig. 5, including the results for uncoupled case, dimensions $d=1$ and 2, and the global coupling (mean field).

As one increases further the coupling strength, the ability of the system to lift itself out of the absorbing state, however, again decreases and a reentrant phase transition is found at another larger critical value of $K$, just as in case (b). Within the context of the intuitive arguments given above, this feature can be explained by the fact that the transient increase of $\langle x\rangle$ is preceded by a decrease for very small times: By coupling the units too strongly, each of them can explore only its own unperturbed dynamics for very short times and the decreasing tendency of $\langle x\rangle$ with time prevails. Note that one can explain in the same way the reentrant behavior in the phase diagram for the dichotomous noise case; see Fig. 1.

We finally turn to a further comparison of the mean-field results with those obtained through extensive simulations of Eq. (4) for the periodic modulation in finite-dimensional systems. One finds qualitatively similar phase diagrams, for nearest-neighbor coupling with strength $K_{i j}=K / 2 d$, even in low-dimensional cases $d=1$ and 2. Results in $d=1$ are included in Fig. 2. The location of the transition line is predicted surprisingly well by the mean-field theory. We also performed extensive simulations in the presence of a nonlinearity $-x_{i}^{3}$. The presence of this term does not modify the location of the phase transition, but the transition is now of second order and the order parameter grows continuously from zero as one leaves the absorbing state; see Fig. 6. Furthermore, even though the dynamics are purely deterministic, the statistical properties seems to converge to steady-state values and one can, e.g., obtain accurate results for spatial correlation functions. These results are represented in Fig. 7 [ $d=1$ and 2 for case (c) of periodic modulation]. One recognizes the appearance of long-range spatial correlations in the vicinity of the phase transition.

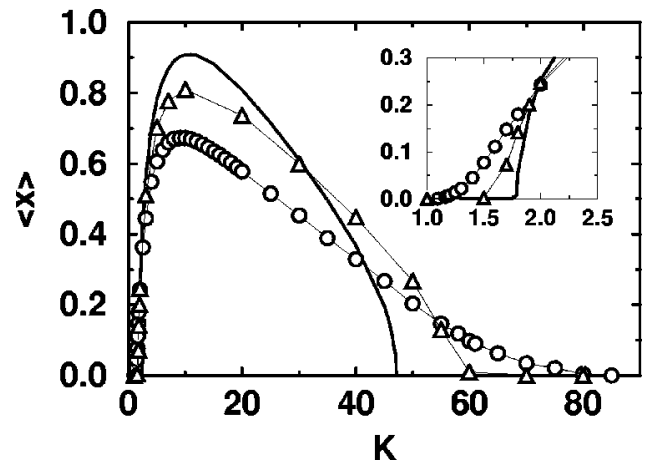

FIG. 6. First moment for the case of periodic modulation ( $\omega=10$ and $A=10$ ) as a function of the coupling constant $K$. The one-dimensional system (circles), two-dimensional system (triangles), and mean-field theory (solid lines) are shown.

\section{ACKNOWLEDGMENTS}

This work was supported by NATO Grant No. CRG 950055. C.V.d.B. also acknowledges support from the Program on Inter-University Attraction Poles, Prime Minister's Office, Belgian Government and NFWO Belgium. R.K. acknowledges support from the Alabama Education and Research Network. Most of the computer simulations were carried out using Cray C90 at the Alabama Supercomputer Center.

\section{APPENDIX}

In cases (a) and (b), aspects of the spatiotemporal intermittency that characterizes the uncoupled system persists not only in finite dimensions [29], but even in the mean-field description. To show this, consider the Gaussian white-noise case (a). The $n$th moments $\mu_{n}=\left\langle x^{n}\right\rangle$ of $P(x, t)$ obey the set of equations

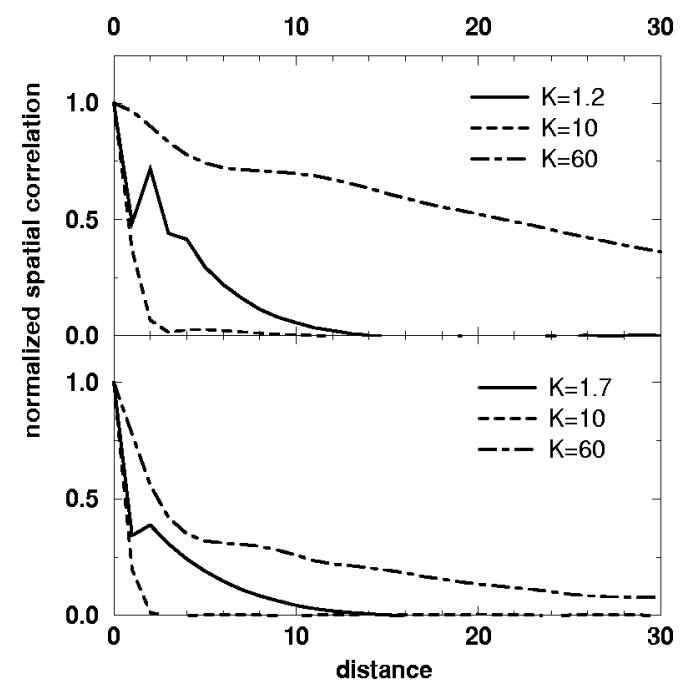

FIG. 7. Spatial correlations for the case of periodic modulation ( $\omega=10$ and $A=10$ ). The upper graph is for the one-dimensional system and the lower graph for the two-dimensional system. Solid and dot-dashed lines represent the vicinities of the first critical point and the reentrant transition point, respectively, and dashed line corresponds to the ordered phase. 


$$
\dot{\mu}_{n}=-n\left(1+K-n \frac{\sigma^{2}}{2}\right) \mu_{n}+n K \mu_{1} \mu_{n-1} .
$$

To extract the asymptotic behavior for $t \rightarrow \infty$ one sets $\mu_{n} \sim \exp \left(a_{n} t\right)$. One then finds that $a_{n}=-n\left(1+K-n \sigma^{2} / 2\right)$ if $a_{n}>-1+\sigma^{2} / 2+a_{n-1}$ and $a_{n}=-1+\sigma^{2} / 2+a_{n-1}$ otherwise. With $a_{1}=-1+\sigma^{2} / 2$, the behavior of all the moments can be extracted. The behavior of the second moment is of particular interest since the very validity of the mean-field descrip- tion depends on it. One finds that $a_{2}=-2\left(1+K-\sigma^{2}\right)$ for $K<\sigma^{2} / 2$ and $a_{2}=-2\left(1-\sigma^{2} / 2\right)=2 a_{1}$ for $K>\sigma^{2} / 2$. We conclude that the second moment $\left\langle x^{2}\right\rangle$ diverges more rapidly than $\langle x\rangle^{2}$ for $K<\sigma^{2} / 2$. As a result, one expects that in any finite system, the relative fluctuations of $(1 / N) \sum_{j=1}^{N} x_{j}(t)$ will become large after a time of the order $t \sim \ln N$ when $K<\sigma^{2} / 2$. For larger times, one will observe large sample to sample fluctuations and the mean-field approximation will completely break down.
[1] A. S. Mikhailov, Phys. Rep. 184, 308 (1989).

[2] Noise in Nonlinear Dynamical Systems, edited by F. Moss and P. V. E. McClintock (Cambridge University Press, Cambridge, 1989).

[3] L. Schimansky-Geier and Ch. Zulicke, Z. Phys. B 82, 157 (1991)

[4] J. García-Ojalvo, A. Hernández-Machado, and J. M. Sancho, Phys. Rev. Lett. 71, 1542 (1993).

[5] L. Ramírez-Piscina, A. Hernández-Machado, and J. M. Sancho, Phys. Rev. B 48, 119 (1993).

[6] C. Van den Broeck, J. M. R. Parrondo, J. Armero, and A. Hernández-Machado, Phys. Rev. E 49, 2639 (1994).

[7] C. Van den Broeck, J. M. R. Parrondo, and R. Toral, Phys. Rev. Lett. 73, 3395 (1994).

[8] A. Becker and L. Kramer, Phys. Rev. Lett. 73, 955 (1994).

[9] A.S. Pikovsky, K. Rateitschak, and J. Kurths, Z. Phys. B 95, 541 (1994).

[10] F. Jülicher and J. Prost, Phys. Rev. Lett. 75, 2618 (1995).

[11] J. M. R. Parrondo, C. Van den Broeck, J. Buceta, and J. de la Rubia, Physica A 224, 153 (1996).

[12] J. García-Ojalvo, J. M. R. Parrondo, J. M. Sancho, and C. Van den Broeck, Phys. Rev. E 54, 6918 (1996).

[13] G. Grinstein, M. A. Munoz, and T. Yuhai, Phys. Rev. Lett. 76, 4376 (1996).

[14] S. H. Park and S. Kim, Phys. Rev. E 53, 3425 (1996).

[15] J.-H. Li and Z.-Q. Huang, Phys. Rev. E 53, 3315 (1996).

[16] C. Van den Broeck, J. M. R. Parrondo, R. Toral, and R. Kawai, Phys. Rev. E 55, 4084 (1997).
[17] S. Kim, S.H. Park, Ch. Doering, and C.S. Ryu, Phys. Lett. A 224, 147 (1997).

[18] R. Muller, K. Lippert, A. Kuhnel, and U. Behn, Phys. Rev. E 56, 2658 (1997).

[19] S. Kim, S. H. Park, and C. S. Ryu, ETRI J. 18, 147 (1996).

[20] J.-H. Li and Z.-Q. Huang (unpublished).

[21] J. Martín-Regalado, S. Balle, and N. B. Abraham, IEEE J. Quantum Electron. 32, 257 (1996).

[22] C. W. Meyer, G. Ahlers, and D. S. Cannell, Phys. Rev. A 44, 2514 (1991).

[23] H. K. Janssen, Z. Phys. B 42, 151 (1981); P. Grassberger, ibid. 47, 365 (1981); T. Vicsek, in Proceedings of the Workshop on Surface Disordering: Growth, Roughening, and Phase Transitions, Proceedings of the Les Houches Summer School of Theoretical Physics, Les Houches, 1992, edited by R. Jullien, J. Kertesz, P. Meakin, and D. E. Wolf (Nova Science, Commack, NY, 1992), p. 155; R. Dickman, Phys. Rev. E 50, 4404 (1994); A. L. Barabasi and H. E. Stanley, Fractal Concepts in Surface Growth (University of Cambridge Press, Cambridge, 1995).

[24] N. G. van Kampen, Stochastic Processes in Chemistry and Physics (North-Holland, Amsterdam, 1981).

[25] R. Graham and A. Schenzle, Phys. Rev. A 25, 1731 (1982).

[26] R.Z. Khas'minksii, Theor. Probab. Appl. 12, 144 (1967).

[27] R.C. Desai and R. Zwanzig, J. Stat. Phys. 19, 1 (1978).

[28] L.L. Bonilla, J. Stat. Phys. 46, 659 (1987).

[29] A. S. Mikhailov and A. Yu. Loskutov, Foundations of Synergetics II (Springer, Berlin, 1996).

[30] S. Mangioni, R. Deza, H. Wio, and R. Toral, Phys. Rev. Lett. 79, 2389 (1997). 